\title{
TWO-INTERVAL EVEN-ORDER DIFFERENTIAL OPERATORS IN MODIFIED HILBERT SPACES
}

\author{
JIANQING SUO and WANYI WANG
}

\author{
(Received 17 April 2011)
}

\begin{abstract}
By modifying the inner product in the direct sum of the Hilbert spaces associated with each of two underlying intervals on which an even-order equation is defined, we generate self-adjoint realisations for boundary conditions with any real coupling matrix which are much more general than the coupling matrices from the 'unmodified' theory.
\end{abstract}

2010 Mathematics subject classification: primary 34B20, 34B24; secondary 47B25.

Keywords and phrases: self-adjoint, interface conditions, transmission conditions.

\section{Introduction}

Partly motivated by applications, in particular [1] (see also [4]), Everitt and Zettl in [2] developed a theory of self-adjoint realisations of Sturm-Liouville problems on two intervals in the direct sum of Hilbert spaces associated with these intervals. See [10, Ch. 13] for an exposition of this theory. This theory was extended in [3] to higher-order regular and singular equations and any number of intervals, finite or infinite.

As in the one-interval case the characterisation of [3] depends on maximal domain vectors. These vectors depend on the coefficients of each differential equation and this dependence is implicit and complicated. In [9] Wang et al. give an explicit characterisation of all self-adjoint domains for singular problems in terms of certain solutions for real $\lambda$ for the one-interval case when one endpoint is regular and the other is singular. In analogy with the celebrated Weyl limit-point (LP), limit-circle (LC) theory in the second-order case, they construct LC and LP solutions and characterise the self-adjoint domains in terms of the LC solutions. Following [9], Hao et al. give a new characterisation in [5] by dividing $\left(a_{1}, b_{1}\right)$ into two intervals $\left(a_{1}, c_{1}\right)$ and $\left(c_{1}, b_{1}\right)$ for some $c_{1} \in\left(a_{1}, b_{1}\right)$ and using the LC solutions on each interval constructed in [9] when $a_{1}$ and $b_{1}$ are singular. In [7], Suo and Wang extend the characterisation in [5] to the two-interval case but the result reduces to the case when one, two, three or four endpoints are regular.

The work of the first two authors is supported by the National Nature Science Foundation of China (grant number 10961019) and the '211 project' innovative talents training program of the Inner Mongolia University.

(C) 2011 Australian Mathematical Publishing Association Inc. 0004-9727/2011 \$16.00 
As noted in [2], a simple way of getting self-adjoint operators in a direct-sum Hilbert space is to take the direct sum of self-adjoint operators from each of the separate Hilbert spaces. However, there are many self-adjoint operators which are not merely the sum of self-adjoint operators from each of the separate intervals. These 'new' self-adjoint operators involve interactions between the two intervals. Therefore in [2] the authors develop a 'two-interval' theory. Mukhtarov and Yakubov [6] observed that the set of two-interval self-adjoint realisations can be further enlarged by using different multiples of the usual inner products associated with each of the intervals. In [8] Sun et al. use the Mukhtarov-Yakubov modification of the Everitt-Zettl theory to characterise all self-adjoint realisations of regular two-interval problems. This characterisation is explicit and involves only the values of solutions and their quasiderivatives at the endpoints of the intervals and the multiple inner product parameters. In particular, for the second-order case with coupled boundary conditions and a real coupling matrix $K$, the method of [2] requires that $\operatorname{det}(K)=1$ whereas with the Mukhtarov-Yakubov modification in [8] it is only required that $\operatorname{det}(K)$ is positive.

In this paper we develop a complete analogue of [7] when one endpoint of each interval $\left(a_{1}, b_{1}\right),\left(a_{2}, b_{2}\right)$ is regular using Hilbert spaces but with the usual inner products replaced by appropriate multiples. The interplay of these multiples with the boundary conditions generates self-adjoint problems of even order with real coupling matrices $K$ which are much more general than the coupling matrices from the 'unmodified' theory. We give a number of examples to illustrate this additional generality, among other things.

From another perspective, instead of using multiples of the usual inner products, our approach can be described as using multiples of weight functions.

\section{Notation and basic facts for one interval}

Although we only consider even-order equations with real coefficients in this paper, we summarise some basic facts about general quasidifferential equations of even and odd order and real or complex coefficients for the convenience of the reader.

Let $J=(a, b)$ be an interval with $-\infty \leq a<b \leq \infty$ and let $n$ be a positive integer (even or odd). For a given set $S, M_{n}(S)$ denotes the set of $n \times n$ complex matrices with entries from $S$.

Let $Z_{n}(J):=\left\{Q=\left(q_{i s}\right)_{i, s=1}^{n}\right\}$, where

$$
\left\{\begin{array}{l}
q_{i, i+1} \neq 0 \text { almost everywhere on } J, \quad q_{i, i+1}^{-1} \in L_{\mathrm{loc}}(J), \quad 1 \leq i \leq n-1, \\
q_{i s}=0 \text { almost everywhere on } J, \quad 2 \leq i+1<s \leq n, \\
q_{i s} \in L_{\mathrm{loc}}(J), \quad s \neq i+1,1 \leq i \leq n-1 .
\end{array}\right.
$$

Let $Q \in Z_{n}(J)$. We define

$$
V_{0}:=\{y: J \rightarrow \mathbb{C}, y \text { is measurable }\}
$$


and

$$
y^{[0]}:=y \quad\left(y \in V_{0}\right)
$$

Inductively, for $i=1, \ldots, n$, we define

$$
\begin{gathered}
V_{i}=\left\{y \in V_{i-1}: y^{[i-1]} \in A C_{\mathrm{loc}}(J)\right\}, \\
y^{[i]}=q_{i, i+1}^{-1}\left(y^{[i-1]^{\prime}}-\sum_{s=1}^{i} q_{i s} y^{[s-1]}\right) \quad\left(y \in V_{i}\right),
\end{gathered}
$$

where $q_{n, n+1}:=1$, and $A C_{\text {loc }}(J)$ denotes the set of complex-valued functions which are absolutely continuous on all compact subintervals of $J$. Finally we set

$$
M y=M_{Q} y:=i^{n} y^{[n]} \quad\left(y \in V_{n}\right) .
$$

The expression $M=M_{Q}$ is called the quasidifferential expression associated with $Q$. For $V_{n}$ we also write $V(M)$ and $D(Q)$. The function $y^{[i]}(0 \leq i \leq n)$ is called the $i$ th quasiderivative of $y$. Since the quasiderivative depends on $Q$, we sometimes write $y_{Q}^{[i]}$ instead of $y^{[i]}$.

REMARK 2.1. The operator $M: D(Q) \rightarrow L_{\mathrm{loc}}(J)$ is linear.

Let $Z_{n}(J, \mathbb{R})$ denote the set of matrices $Q \in Z_{n}(J)$ which have real-valued components.

Definition 2.2. Let $Q \in Z_{n}(J, \mathbb{R})$ and let $M=M_{Q}$ be defined as above. Assume that

$$
Q=-E^{-1} Q^{*} E, \quad \text { where } E=\left((-1)^{i} \delta_{i, n+1-s}\right)_{i, s=1}^{n} .
$$

Then $M=M_{Q}$ is called a symmetric differential expression.

Let $w \in L_{\mathrm{loc}}(J)$ be positive almost everywhere on $J$. We consider the Hilbert space

$$
H=L^{2}(J, w)
$$

with its usual inner product

$$
\langle y, v\rangle_{w}:=\int_{J} y \bar{v} w .
$$

The maximal and minimal operators associated with a symmetric expression $Q$ and a positive weight function $w$ in the Hilbert space $H$ are defined as follows.

Definition 2.3. Assume $Q \in Z_{n}(J, \mathbb{R})$ satisfies (2.2) and let $M=M_{Q}$ be the associated symmetric expression. Let $w \in L_{\mathrm{loc}}(J)$ be positive almost everywhere on $J$. Define

$$
\begin{gathered}
D_{\max }=\left\{y \in L^{2}(J, w): y \in D(Q), w^{-1} M y \in L^{2}(J, w)\right\}, \\
S_{\max } y=w^{-1} M y, \quad y \in D_{\max }, \\
S_{\min }=S_{\max }^{*}, \\
D_{\min }=D\left(S_{\min }\right) .
\end{gathered}
$$


Lemma 2.4. Let $S_{\min }$ and $S_{\max }$ be defined as above. Then $D_{\min }$ and $D_{\max }$ are dense in $H, S_{\min }$ and $S_{\max }$ are closed operators in $H, S_{\min }^{*}=S_{\max }, S_{\min }=S_{\max }^{*}$ and $S_{\min }$ is a symmetric operator in $H$.

From Definition 2.3 and Lemma 2.4 we see that every self-adjoint extension $S$ of the minimal operator is 'between' the minimal and maximal operators; that is,

$$
S_{\text {min }} \subset S=S^{*} \subset S_{\text {max }} .
$$

Thus these self-adjoint operators $S$ are distinguished from one another only by their domains.

Lemma 2.5 (Lagrange identity). Assume $Q \in Z_{n}(J, \mathbb{R})$ satisfies (2.2) and let $M=M_{Q}$ be the corresponding differential expression. Then, for any $y, z \in D(Q)$,

$$
\bar{z} M y-y \overline{M z}=[y, z]^{\prime},
$$

where

$$
\begin{gathered}
{[y, z]=(-1)^{k} \sum_{i=0}^{n-1}(-1)^{n+1-i} \bar{z}^{[n-i-1]} y^{[i]}=(-1)^{k}\left(Z^{*} E Y\right),} \\
Y=\left(\begin{array}{c}
y \\
y^{[1]} \\
\vdots \\
y^{[n-1]}
\end{array}\right), \quad Z=\left(\begin{array}{c}
z \\
z^{[1]} \\
\vdots \\
z^{[n-1]}
\end{array}\right) .
\end{gathered}
$$

Definition 2.6 (Regular endpoints). Let $Q \in Z_{n}(J, \mathbb{R}), J=(a, b)$. The expression $M=M_{Q}$ is said to be regular at $a$ if for some $c, a<c<b$, we have

$$
\begin{gathered}
q_{i, i+1}^{-1} \in L(a, c), \quad i=1, \ldots, n-1 ; \\
q_{i s} \in L(a, c), \quad 1 \leq i, s \leq n, s \neq i+1 .
\end{gathered}
$$

Similarly the endpoint $b$ is regular if for some $c, a<c<b$, we have

$$
\begin{gathered}
q_{i, i+1}^{-1} \in L(c, b), \quad i=1, \ldots, n-1 ; \\
q_{i s} \in L(c, b), \quad 1 \leq i, s \leq n, s \neq i+1 .
\end{gathered}
$$

Note that, from (2.1) it follows that if the above hold for some $c \in J$ then they hold for any $c \in J$. We say that $M$ is regular on $J$, or just $M$ is regular, if $M$ is regular at both endpoints.

\section{Notation and Basic Assumptions for Two Intervals}

Let

$$
J_{r}=\left(a_{r}, b_{r}\right), \quad-\infty<a_{r}<b_{r} \leq \infty, r=1,2 .
$$

Define two differential expressions with real-valued coefficients by

$$
M_{r} y=M_{Q_{r}} y:=i^{n} y^{[n]} \quad \text { on } J_{r}, r=1,2, n=2 k, k>1 .
$$


Let

$$
H_{r}=L^{2}\left(J_{r}, w_{r}\right), \quad w_{r}>0, r=1,2 .
$$

The two-interval maximal and minimal domains and operators are simply the direct sums of the corresponding one-interval domains and operators:

$$
\begin{array}{cc}
D_{\max }=D_{1 \max }+D_{2 \max }, & D_{\text {min }}=D_{1 \min }+D_{2 \min }, \\
S_{\text {max }}=S_{1 \max }+S_{2 \max }, & S_{\text {min }}=S_{1 \min }+S_{2 \min .}
\end{array}
$$

Elements of $H_{u}=H_{1}+H_{2}$ will be denoted in bold face type: $\mathbf{f}=\left\{f_{1}, f_{2}\right\}$ with $f_{1} \in H_{1}$, $f_{2} \in H_{2}$. As usual the inner product in $H_{u}$ is defined by

$$
(\mathbf{f}, \mathbf{g})=\left(f_{1}, g_{1}\right)_{1}+\left(f_{2}, g_{2}\right)_{2},
$$

where $(\cdot, \cdot)_{r}$ is the usual inner product in $H_{r}$ :

$$
\left(f_{r}, g_{r}\right)_{r}=\int_{J_{r}} f_{r} \bar{g}_{r} w_{r} .
$$

In this paper, following [8] we replace the direct-sum inner product (3.1) by

$$
\langle\mathbf{f}, \mathbf{g}\rangle=l\left(f_{1}, g_{1}\right)_{1}+s\left(f_{2}, g_{2}\right)_{2}, \quad l>0, s>0,
$$

and apply operator theory in the direct-sum space

$$
H=\left(L^{2}\left(J_{1}, w_{1}\right) \dot{+} L^{2}\left(J_{2}, w_{2}\right),\langle\cdot, \cdot\rangle\right) .
$$

Remark 3.1. Note that (3.2) is an inner product in $H$ for any positive numbers $l$ and $s$. The elements of the Hilbert space $H$ defined by (3.3) are the same as those of the usual direct-sum Hilbert spaces $H_{u}$; thus these spaces are differentiated from each other only by their inner products. As we will see below, the parameters $l, s$ influence the boundary conditions which yield self-adjoint realisations of the equations in the two-interval case. Observe also that the Hilbert space (3.3) can be viewed as a 'usual' direct-sum space $H_{u}$ with summands $H_{r}=L^{2}\left(J_{r}, w_{r}\right)$ but with each $w_{r}$ replaced by an appropriate multiple.

As in the one-interval case the Lagrange sesquilinear form plays an important role. It is defined, for appropriate functions $\mathbf{f}, \mathbf{g}$, by

$$
[\mathbf{f}, \mathbf{g}]=l\left[f_{1}, g_{1}\right]_{1}\left(b_{1}\right)-l\left[f_{1}, g_{1}\right]_{1}\left(a_{1}\right)+s\left[f_{2}, g_{2}\right]_{2}\left(b_{2}\right)-s\left[f_{2}, g_{2}\right]_{2}\left(a_{2}\right),
$$

where

$$
\left[f_{r}, g_{r}\right]_{r}=(-1)^{k}\left(G_{r}^{*} E F_{r}\right), \quad F_{r}=\left(\begin{array}{c}
f_{r} \\
f_{r}^{[1]} \\
\vdots \\
f_{r}^{[n-1]}
\end{array}\right), \quad G_{r}=\left(\begin{array}{c}
g_{r} \\
g_{r}^{[1]} \\
\vdots \\
g_{r}^{[n-1]}
\end{array}\right) .
$$

Note that the two-interval Lagrange form $[f, g]$ connects all four endpoints with each other and depends on the parameters $l, s$. 


\section{Characterisation of all self-adjoint domains for two-interval problems}

In this section we assume that $M_{Q_{r}}(r=1,2)$ are generated by $Q_{r} \in Z_{n(r)}\left(J_{r}, \mathbb{R}\right)$ satisfying (2.2), $n=2 k, k>1$. First, we give some preliminary lemmas.

\section{Lemma 4.1.}

(1) The following equalities hold.

$$
\begin{aligned}
& S_{\text {min }}^{*}=S_{1 \text { min }}^{*}+S_{2 \min }^{*}=S_{1 \max }+S_{2 \max }=S_{\text {max }}, \\
& S_{\text {max }}^{*}=S_{1 \text { max }}^{*}+S_{2 \max }^{*}=S_{1 \text { min }}+S_{2 \min }=S_{\text {min }} .
\end{aligned}
$$

In particular,

$$
\begin{gathered}
D_{\text {max }}=D\left(S_{\text {max }}\right)=D\left(S_{1 \max }\right)+D\left(S_{2 \max }\right), \\
D_{\text {min }}=D\left(S_{\text {min }}\right)=D\left(S_{1 \text { min }}\right)+D\left(S_{2 \min }\right) .
\end{gathered}
$$

(2) The minimal operator $S_{\min }$ is a closed, symmetric, densely defined operator in the Hilbert space $H$ with deficiency index d given by $d=d_{1}+d_{2}$.

Proof. The proof given in [2] for (3.1) extends readily to (3.2).

Definition 4.2. Assume that the endpoint $a_{r}$ is regular and $S_{r \min }$ is defined as above. Then for each $r$ the deficiency index $d_{r}$ of $S_{r \min }$ is the number of linearly independent solutions of

$$
M_{r} y=i w_{r} y \quad \text { on } J_{r}, \quad i=\sqrt{-1}, r=1,2
$$

which lie in $H_{r}$.

Lemma 4.3. The number $d_{r}$ of linearly independent solutions of

$$
M_{r} y=\lambda_{r} w_{r} y \quad \text { on } J_{r}, \quad r=1,2,
$$

lying in $H_{r}$ is independent of $\lambda_{r} \in \mathbb{C}$, provided $\operatorname{Im}\left(\lambda_{r}\right) \neq 0$. The inequalities

$$
k \leq d_{r} \leq 2 k=n
$$

hold. For $\lambda=\lambda_{r} \in \mathbb{R}$, the number of linearly independent solutions of $(4.1)_{r=1}$ is less than or equal to $d_{1}$, and the number of linearly independent solutions of $(4.1)_{r=2}$ is less than or equal to $d_{2}$.

Proof. For the proof of the statement in the last sentence see [9, Lemma 5]. The other statements are well known.

Lemma 4.4. Suppose $M_{r}$ is regular at $c_{r}$. Then for any $y=\left\{y_{1}, y_{2}\right\} \in D_{\max }$ the limits

$$
y_{1}^{[i]}\left(c_{1}\right)=\lim _{t \rightarrow c_{1}} y^{[i]}(t), \quad y_{2}^{[i]}\left(c_{2}\right)=\lim _{t \rightarrow c_{2}} y^{[i]}(t)
$$

exist and are finite. In particular, this holds at any regular endpoint and at each interior point of $J_{r}$. At an endpoint the limit is the appropriate one-sided limit.

Proof. This follows from the one-interval theory, see [9, Lemma 3]. 
Lemma 4.5 (Naimark patching lemma). Let $Q_{r} \in Z_{n(r)}\left(J_{r}, \mathbb{R}\right)$ and assume that $M_{r}$ is regular on $J_{r}$. Suppose that $w_{r} \in L\left(J_{r}\right), w_{r}>0$ on $J_{r}, r=1,2$. Let $\alpha_{s}, \beta_{s}, \delta_{s}, \eta_{s} \in \mathbb{C}$, $s=0, \ldots, n-1$. Then there is a function $y=\left\{y_{1}, y_{2}\right\} \in D_{\max }$ such that

$$
y_{1}^{[s]}\left(a_{1}\right)=\alpha_{s}, \quad y_{1}^{[s]}\left(b_{1}\right)=\beta_{s}, \quad y_{2}^{[s]}\left(a_{2}\right)=\delta_{s}, \quad y_{2}^{[s]}\left(b_{2}\right)=\eta_{s} \quad(s=0, \ldots, n-1) .
$$

Proof. This follows from the one-interval theory; see [9, Lemma 4].

Corollary 4.6. Let $c_{r}<d_{r} \in J_{r}, r=1,2$, and $\alpha_{s}, \beta_{s}, \delta_{s}, \eta_{s} \in \mathbb{C}, s=0, \ldots, n-1$. Then there is a $y=\left\{y_{1}, y_{2}\right\} \in D_{\max }$ such that $y_{r}$ has compact support in $J_{r}$ and satisfies

$$
y_{1}^{[s]}\left(c_{1}\right)=\alpha_{s}, \quad y_{1}^{[s]}\left(d_{1}\right)=\beta_{s}, \quad y_{2}^{[s]}\left(c_{2}\right)=\delta_{s}, \quad y_{2}^{[s]}\left(d_{2}\right)=\eta_{s} \quad(s=0, \ldots, n-1) .
$$

Proof. This follows from the one-interval theory; see [9, Corollary 4].

COROLlaRy 4.7. Let $a_{1 r}<\cdots<a_{k r} \in J_{r}, r=1,2$, where $a_{1 r}$ and $a_{k r}$ can also be regular endpoints. Let $\alpha_{j s}, \beta_{j s} \in \mathbb{C}, j=1, \ldots, k, s=0, \ldots, n-1$. Then there is a $y=\left\{y_{1}, y_{2}\right\} \in$ $D_{\max }$ such that

$$
y_{1}^{[s]}\left(a_{j 1}\right)=\alpha_{j s}, \quad y_{2}^{[s]}\left(a_{j 2}\right)=\beta_{j s} \quad(j=1, \ldots, k, s=0, \ldots, n-1) .
$$

PRoof. This follows from repeated applications of the previous corollary.

Lemma 4.8. Suppose $a_{r}, r=1,2$ is regular. Then minimal domains $D_{\min }$ consist of all functions $y=\left\{y_{1}, y_{2}\right\} \in D_{\max }$ which satisfy the following two conditions.

(1) $y_{1}^{[j]}\left(a_{1}\right)=y_{2}^{[j]}\left(a_{2}\right)=0, j=0,1, \ldots, n-1$.

(2) For all $z=\left\{z_{1}, z_{2}\right\} \in D_{\max }$,

$$
\left[y_{1}, z_{1}\right]_{1}\left(b_{1}\right)=\left[y_{2}, z_{2}\right]_{2}\left(b_{2}\right)=0 .
$$

Proof. This follows from the one-interval theory; see [9, Lemma 7]. A similar result holds when the endpoints $b_{r}$ are regular.

Next we give the decomposition of the maximal domain and the characterisation of all self-adjoint domains for the case where $a_{1}$ and $a_{2}$ are regular.

Let

$$
a_{1}<c<b_{1}, \quad a_{2}<d<b_{2},
$$

and determine functions $z_{j} \in D_{1 \max }, g_{j} \in D_{2 \max }, j=1, \ldots, n$, such that $z_{j}(t)=0$ for $t \geq c$ and $g_{j}(t)=0$ for $t \geq d, j=1, \ldots, n$, and

$$
\left(\begin{array}{ccccc}
{\left[z_{1}, z_{1}\right]_{1}\left(a_{1}\right)} & {\left[z_{2}, z_{1}\right]_{1}\left(a_{1}\right)} & \cdots & {\left[z_{n-1}, z_{1}\right]_{1}\left(a_{1}\right)} & {\left[z_{n}, z_{1}\right]_{1}\left(a_{1}\right)} \\
{\left[z_{1}, z_{2}\right]_{1}\left(a_{1}\right)} & {\left[z_{2}, z_{2}\right]_{1}\left(a_{1}\right)} & \cdots & {\left[z_{n-1}, z_{2}\right]_{1}\left(a_{1}\right)} & {\left[z_{n}, z_{2}\right]_{1}\left(a_{1}\right)} \\
\vdots & \vdots & \vdots & \vdots & \vdots \\
{\left[z_{1}, z_{n}\right]_{1}\left(a_{1}\right)} & {\left[z_{2}, z_{n}\right]_{1}\left(a_{1}\right)} & \cdots & {\left[z_{n-1}, z_{n}\right]_{1}\left(a_{1}\right)} & {\left[z_{n}, z_{n}\right]_{1}\left(a_{1}\right)}
\end{array}\right)
$$




$$
\begin{aligned}
& =\left(\begin{array}{rrrrr}
0 & 0 & \cdots & 0 & -1 \\
0 & \cdots & 0 & 1 & 0 \\
\vdots & & . & & \vdots \\
0 & -1 & 0 & \cdots & 0 \\
1 & 0 & \cdots & 0 & 0
\end{array}\right)=E, \\
& \left(\begin{array}{ccccc}
{\left[g_{1}, g_{1}\right]_{2}\left(a_{2}\right)} & {\left[g_{2}, g_{1}\right]_{2}\left(a_{2}\right)} & \cdots & {\left[g_{n-1}, g_{1}\right]_{2}\left(a_{2}\right)} & {\left[g_{n}, g_{1}\right]_{2}\left(a_{2}\right)} \\
{\left[g_{1}, g_{2}\right]_{2}\left(a_{2}\right)} & {\left[g_{2}, g_{2}\right]_{2}\left(a_{2}\right)} & \cdots & {\left[g_{n-1}, g_{2}\right]_{2}\left(a_{2}\right)} & {\left[g_{n}, g_{2}\right]_{2}\left(a_{2}\right)} \\
\vdots & \vdots & \vdots & \vdots & \vdots \\
{\left[g_{1}, g_{n}\right]_{2}\left(a_{2}\right)} & {\left[g_{2}, g_{n}\right]_{2}\left(a_{2}\right)} & \cdots & {\left[g_{n-1}, g_{n}\right]_{2}\left(a_{2}\right)} & {\left[g_{n}, g_{n}\right]_{2}\left(a_{2}\right)}
\end{array}\right) \\
& =\left(\begin{array}{rrrrr}
0 & 0 & \cdots & 0 & -1 \\
0 & \cdots & 0 & 1 & 0 \\
\vdots & & . & & \vdots \\
0 & -1 & 0 & \cdots & 0 \\
1 & 0 & \cdots & 0 & 0
\end{array}\right)=E .
\end{aligned}
$$

Such functions exist by the patching lemma and the fact that for each $i=1, \ldots, n$ the values $z_{i}^{[j]}\left(a_{1}\right)$ and $g_{i}^{[j]}\left(a_{2}\right)$ can be assigned arbitrarily.

THEOREM 4.9. Let the endpoints $a_{r}$ be regular while the endpoints $b_{r}$ are singular, let $d_{r}$ be the deficiency index of the minimal operator $S_{r \min }$, and let $m_{r}=2 d_{r}-2 k, r=1,2$. Assume there exists $\lambda_{1}$ and $\lambda_{2} \in \mathbb{R}$ such that (4.1) $r=1$ has $d_{1}$ linearly independent solutions lying in $H_{1}$ and $(4.1)_{r=2}$ has $d_{2}$ linearly independent solutions lying in $H_{2}$. Then there exist solutions $u_{j}, j=1, \ldots, m_{1}$, of $(4.1)_{r=1}$ lying in $H_{1}$ and $v_{j}$, $j=1, \ldots, m_{2}$, of $(4.1)_{r=2}$ lying in $H_{2}$ such that the $m_{1} \times m_{1}$ and $m_{2} \times m_{2}$ matrices

$$
U=\left(\left[u_{i}, u_{j}\right]_{1}\left(a_{1}\right)\right), \quad 1 \leq i, j \leq m_{1}, \quad V=\left(\left[v_{i}, v_{j}\right]_{2}\left(a_{2}\right)\right), \quad 1 \leq i, j \leq m_{2},
$$

are nonsingular and

$$
\begin{aligned}
& D_{1 \max }=D_{1 \min } \dot{+} \operatorname{span}\left\{z_{1}, z_{2}, \ldots, z_{n}\right\} \dot{+} \operatorname{span}\left\{u_{1}, u_{2}, \ldots, u_{m_{1}}\right\}, \\
& D_{2 \max }=D_{2 \text { min }} \dot{+} \operatorname{span}\left\{g_{1}, g_{2}, \ldots, g_{n}\right\} \dot{+} \operatorname{span}\left\{v_{1}, v_{2}, \ldots, v_{m_{2}}\right\} .
\end{aligned}
$$

Proof. This follows from the one-interval case; see [9, Theorem 3].

Corollary 4.10. Assume that $a_{r}$ is regular, $r=1$, 2. Let $d_{r}$ be the deficiency index of the minimal operator $S_{r \min }$ and let $m_{r}=2 d_{r}-2 k, r=1,2$. Assume that there exist $\lambda_{1}$ and $\lambda_{2} \in \mathbb{R}$ such that (4.1) $r=1$ has $d_{1}$ linearly independent solutions lying in $H_{1}$ and $(4.1)_{r=2}$ has $d_{2}$ linearly independent solutions lying in $H_{2}$. Then there exist $d_{1}$ and $d_{2}$ linearly independent real solutions $u_{1}, \ldots, u_{d_{1}}$ of $(4.1)_{r=1}$ and $v_{1}, \ldots, v_{d_{2}}$ of $(4.1)_{r=2}$ for $\lambda_{1}$ and $\lambda_{2}$ satisfying the following three conditions.

(1) The $m_{1} \times m_{1}$ and $m_{2} \times m_{2}$ matrices

$$
U=\left(\left[u_{i}, u_{j}\right]_{1}\left(a_{1}\right)\right), \quad 1 \leq i, j \leq m_{1}, \quad V=\left(\left[v_{i}, v_{j}\right]_{2}\left(a_{2}\right)\right), \quad 1 \leq i, j \leq m_{2},
$$


are given by

$$
\begin{aligned}
& U=(-1)^{k+1} E_{m_{1} \times m_{1}}, \\
& V=(-1)^{k+1} E_{m_{2} \times m_{2}},
\end{aligned}
$$

and are therefore nonsingular.

(2) For every $y_{r} \in D_{r \max }$,

$$
\begin{array}{cc}
{\left[u_{j}, y_{1}\right]_{1}\left(b_{1}\right)=0} & \text { for } j=m_{1}+1, \ldots, d_{1}, \\
{\left[v_{j}, y_{2}\right]_{2}\left(b_{2}\right)=0} & \text { for } j=m_{2}+1, \ldots, d_{2} .
\end{array}
$$

(3) Further,

$$
\begin{array}{cl}
{\left[u_{i}, u_{j}\right]_{1}\left(a_{1}\right)=\left[u_{i}, u_{j}\right]_{1}\left(b_{1}\right)=0} & \text { for } i=1, \ldots, d_{1}, j=m_{1}+1, \ldots, d_{1}, \\
{\left[v_{i}, v_{j}\right]_{2}\left(a_{2}\right)=\left[v_{i}, v_{j}\right]_{2}\left(b_{2}\right)=0} & \text { for } i=1, \ldots, d_{2}, j=m_{2}+1, \ldots, d_{2} .
\end{array}
$$

Proof. This follows from the one-interval case; see [9, Corollary 6].

Next we give the Everitt and Zettl [3] extension of the Glazman, Krein, Naimark theorem from the one-interval to the two-interval case.

Lemma 4.11. Let $S_{\min }$ be the two-interval minimal operator in $H$ and let $d$ be the deficiency index of $S_{\min }$. A linear submanifold $D(S)$ of $D_{\max }$ is the domain of a selfadjoint extension $S$ of $S_{\min }$ if and only if there exist vectors $\mathbf{w}_{1}, \mathbf{w}_{2}, \ldots, \mathbf{w}_{d}$ in $D_{\max }$ satisfying the following conditions.

(i) $\mathbf{w}_{1}, \mathbf{w}_{2}, \ldots, \mathbf{w}_{d}$ are linearly independent modulo $D_{\min }$.

(ii) $\quad\left[\mathbf{w}_{i}, \mathbf{w}_{j}\right]=l\left[w_{i 1}, w_{j 1}\right]_{1}\left(b_{1}\right)-l\left[w_{i 1}, w_{j 1}\right]_{1}\left(a_{1}\right)+s\left[w_{i 2}, w_{j 2}\right]_{2}\left(b_{2}\right)-s\left[w_{i 2}, w_{j 2}\right]_{2}\left(a_{2}\right)$ $=0, i, j=1, \ldots, d$.

(iii) $D(S)=\left\{\mathbf{y}=\left\{y_{1}, y_{2}\right\} \in D_{\max }:\left[\mathbf{y}, \mathbf{w}_{j}\right]=l\left[y_{1}, w_{j 1}\right]_{1}\left(b_{1}\right)-l\left[y_{1}, w_{j 1}\right]_{1}\left(a_{1}\right)+s\left[y_{2}\right.\right.$, $\left.\left.w_{j 2}\right]_{2}\left(b_{2}\right)-s\left[y_{2}, w_{j 2}\right]_{2}\left(a_{2}\right)=0, j=1, \ldots, d\right\}$.

Proof. See Theorem 3.1 and Corollary 3.3 in Everitt and Zettl [3] for the case with inner product (3.1); the adaptation to inner product (3.2) is routine.

REMARK 4.12. As mentioned in the Introduction, the characterisation of Lemma 4.11 depends on the maximal domain vectors $w_{j}, j=1, \ldots, d$. These vectors depend on the coefficients of each differential equation and this dependence is implicit and complicated. Based on Theorem 4.9, we next give more explicit equivalent conditions for (i)-(iii) of Lemma 4.11.

The next theorem is our main result in this paper.

THeOREM 4.13. Assume that $a_{r}$ is regular, $r=1,2$. Let $d_{r}$ be the deficiency index of the minimal operator $S_{r \min }$ and let $m_{r}=2 d_{r}-2 k, r=1,2$. Let the Lagrange form $[\cdot, \cdot \cdot]$ be given by (3.4). Assume there exist $\lambda_{1}$ and $\lambda_{2} \in \mathbb{R}$ such that (4.1) $)_{r=1}$ has $d_{1}$ linearly independent solutions lying in $H_{1}$ and $(4.1)_{r=2}$ has $d_{2}$ linearly independent solutions lying in $H_{2}$. Then there exist $d_{1}$ linearly independent real-valued solutions in $H_{1}$ and $d_{2}$ linearly independent real-valued solutions in $H_{2}$. A linear submanifold $D(S)$ of $D_{\max }$ 
is the domain of a self-adjoint extension $S$ of $S_{\min }$ if and only if there exist two complex $d \times n$ matrices $A_{1}$ and $A_{2}$ and two complex $d \times m_{1}$ and $d \times m_{2}$ matrices $B_{1}$ and $B_{2}$ such that the following three conditions hold.

(1) $\operatorname{rank}\left(A_{1}, B_{1}, A_{2}, B_{2}\right)=d$.

(2) $s A_{1} E_{n} A_{1}^{*}-s B_{1} E_{m_{1}} B_{1}^{*}+l A_{2} E_{n} A_{2}^{*}-l B_{2} E_{m_{2}} B_{2}^{*}=0$.

(3) $D(S)=\left\{y=\left\{y_{1}, y_{2}\right\} \in D_{\max }\right\}$, where

$$
\begin{aligned}
& A_{1}\left(\begin{array}{c}
y_{1}\left(a_{1}\right) \\
\vdots \\
y_{1}^{[n-1]}\left(a_{1}\right)
\end{array}\right)+B_{1}\left(\begin{array}{c}
{\left[y_{1}, u_{1}\right]_{1}\left(b_{1}\right)} \\
\vdots \\
{\left[y_{1}, u_{m_{1}}\right]_{1}\left(b_{1}\right)}
\end{array}\right) \\
& +A_{2}\left(\begin{array}{c}
y_{2}\left(a_{2}\right) \\
\vdots \\
y_{2}^{[n-1]}\left(a_{2}\right)
\end{array}\right)+B_{2}\left(\begin{array}{c}
{\left[y_{2}, v_{1}\right]_{2}\left(b_{2}\right)} \\
\vdots \\
{\left[y_{2}, v_{m_{2}}\right]_{2}\left(b_{2}\right)}
\end{array}\right)=\left(\begin{array}{c}
0 \\
\vdots \\
0
\end{array}\right) .
\end{aligned}
$$

Proof. Necessity. Let $D(S)$ be the domain of a self-adjoint extension $S$ of $S_{\min }$. By Lemma 4.11 there exist $w_{1}=\left\{w_{11}, w_{12}\right\}, \ldots, w_{d}=\left\{w_{d 1}, w_{d 2}\right\} \in D_{\max }$ satisfying the conditions (i)-(iii) of Lemma 4.11. By Theorem 4.9, each $w_{i 1}$ and $w_{i 2}$ can be uniquely written as

$$
\begin{aligned}
& w_{i 1}=\widehat{y}_{i 1}+\sum_{s=1}^{n} \tau_{i s} z_{s}+\sum_{j=1}^{m_{1}} e_{i j} u_{j}, \\
& w_{i 2}=\widehat{y}_{i 2}+\sum_{s=1}^{n} \sigma_{i s} g_{s}+\sum_{j=1}^{m_{2}} h_{i j} v_{j},
\end{aligned}
$$

where $\widehat{y}_{i 1} \in D_{1 \min }, \widehat{y}_{i 2} \in D_{2 \min }, \tau_{i j}, e_{i j}, \sigma_{i j}, h_{i j} \in \mathbb{C}$. From (2.3),

$$
\left(\left[y_{r}, w_{j r}\right]_{r}\left(a_{r}\right)\right)_{d \times 1}=(-1)^{k} V_{r}^{*} E_{n}\left(\begin{array}{c}
y_{r}\left(a_{r}\right) \\
\vdots \\
y_{r}^{[n-1]}\left(a_{r}\right)
\end{array}\right),
$$

where

$$
\begin{aligned}
& V_{r}^{*}=\left(\begin{array}{ccc}
\bar{w}_{1 r}\left(a_{r}\right) & \ldots & \bar{w}_{1 r}^{[n-1]}\left(a_{r}\right) \\
\cdots & \cdots & \cdots \\
\bar{w}_{d r}\left(a_{r}\right) & \cdots & \bar{w}_{d r}^{[n-1]}\left(a_{r}\right)
\end{array}\right), \quad r=1,2, \\
& \left(\left[y_{1}, w_{i 1}\right]_{1}\left(b_{1}\right)\right)_{d \times 1}=\left(\left[y_{1}, \widehat{y}_{i 1}+\sum_{s=1}^{n} \tau_{i s} z_{s}+\sum_{j=1}^{m_{1}} e_{i j} u_{j}\right]_{1}\left(b_{1}\right)\right)_{d \times 1} \\
& =\left(\bar{e}_{i j}\right)_{d \times m_{1}}\left(\left[y_{1}, u_{j}\right]_{1}\left(b_{1}\right)\right)_{m_{1} \times 1} \text {, } \\
& \left(\left[y_{2}, w_{i 2}\right]_{2}\left(b_{2}\right)\right)_{d \times 1}=\left(\left[y_{2}, \widehat{y}_{i 2}+\sum_{s=1}^{n} \sigma_{i s} g_{s}+\sum_{j=1}^{m_{2}} h_{i j} v_{j}\right]_{2}\left(b_{2}\right)\right)_{d \times 1} \\
& =\left(\bar{h}_{i j}\right)_{d \times m_{2}}\left(\left[y_{2}, v_{j}\right]_{2}\left(b_{2}\right)\right)_{m_{2} \times 1} \text {. }
\end{aligned}
$$


Let

$$
A_{1}=(-1)^{k+1} l V_{1}^{*} E_{n}, \quad B_{1}=l\left(\bar{e}_{i j}\right)_{d \times m_{1}}, \quad A_{2}=(-1)^{k+1} s V_{2}^{*} E_{n}, \quad B_{2}=s\left(\bar{h}_{i j}\right)_{d \times m_{2}} .
$$

Hence the boundary condition (iii) is equivalent to part (3) of Theorem 4.13.

Next we prove that $A_{1}, B_{1}, A_{2}, B_{2}$ satisfy the conditions (1) and (2) of Theorem 4.13.

Clearly $\operatorname{rank}\left(A_{1}, B_{1}, A_{2}, B_{2}\right) \leq d$. If $\operatorname{rank}\left(A_{1}, B_{1}, A_{2}, B_{2}\right)<d$, then there exist constants $h_{1}, \ldots, h_{d}$, not all zero, such that

$$
\left(h_{1} \cdots h_{d}\right)\left(A_{1}, B_{1}, A_{2}, B_{2}\right)=0 .
$$

Hence $\left(h_{1} \cdots h_{d}\right) A_{1}=(-1)^{k+1}\left(h_{1} \cdots h_{d}\right) l V_{1}^{*} E_{n}=0$ and

$$
\left(h_{1} \cdots h_{d}\right) A_{2}=(-1)^{k+1}\left(h_{1} \cdots h_{d}\right) s V_{2}^{*} E_{n}=0 .
$$

Note that, since $E$ is nonsingular and $l>0, s>0$,

$$
V_{1}\left(\begin{array}{c}
\bar{h}_{1} \\
\vdots \\
\bar{h}_{d}
\end{array}\right)=0, \quad V_{2}\left(\begin{array}{c}
\bar{h}_{1} \\
\vdots \\
\bar{h}_{d}
\end{array}\right)=0
$$

Let $f=\left\{f_{1}, f_{2}\right\}=\sum_{i=1}^{d} \bar{h}_{i} w_{i}$, that is, $f_{1}=\sum_{i=1}^{d} \bar{h}_{i} w_{i 1}, f_{2}=\sum_{i=1}^{d} \bar{h}_{i} w_{i 2}$. Then

$$
\left(\begin{array}{c}
f_{1}\left(a_{1}\right) \\
\vdots \\
f_{1}^{[n-1]}\left(a_{1}\right)
\end{array}\right)=V_{1}\left(\begin{array}{c}
\bar{h}_{1} \\
\vdots \\
\bar{h}_{d}
\end{array}\right)=0, \quad\left(\begin{array}{c}
f_{2}\left(a_{2}\right) \\
\vdots \\
f_{2}^{[n-1]}\left(a_{2}\right)
\end{array}\right)=V_{2}\left(\begin{array}{c}
\bar{h}_{1} \\
\vdots \\
\bar{h}_{d}
\end{array}\right)=0 .
$$

From (4.2),

$$
f_{1}=\sum_{i=1}^{d} \bar{h}_{i} \widehat{y}_{i 1}+\sum_{i=1}^{d} \sum_{s=1}^{n} \bar{h}_{i} \tau_{i s} z_{s}+\sum_{i=1}^{d} \sum_{j=1}^{m_{1}} \bar{h}_{i} e_{i j} u_{j} .
$$

By (4.3), we have $\left(h_{1} \cdots h_{d}\right) B_{1}=0$. Hence

$$
f_{1}=\sum_{i=1}^{d} \bar{h}_{i} \widehat{y}_{i 1}+\sum_{i=1}^{d} \sum_{s=1}^{n} \bar{h}_{i} \tau_{i s} z_{s}
$$

Similarly,

$$
f_{2}=\sum_{i=1}^{d} \bar{h}_{i} \widehat{y}_{i 2}+\sum_{i=1}^{d} \sum_{s=1}^{n} \bar{h}_{i} \sigma_{i s} g_{s}
$$

For any $y=\left\{y_{1}, y_{2}\right\} \in D_{\max }$,

$$
\left[f_{1}, y_{1}\right]_{1}\left(b_{1}\right)=0, \quad\left[f_{2}, y_{2}\right]_{2}\left(b_{2}\right)=0 .
$$

By (4.4) and (4.5), $f_{1} \in D_{1 \min }, f_{2} \in D_{2}$ min. Thus $f=\left\{f_{1}, f_{2}\right\} \in D_{\min }$. This contradicts the fact that the functions $w_{1}, w_{2}, \ldots, w_{d}$ are linearly independent modulo $D_{\min }$. Therefore $\operatorname{rank}\left(A_{1}, B_{1}, A_{2}, B_{2}\right)=d$. 
Now we verify part (2). By (4.2),

$$
\begin{aligned}
l\left[w_{i 1}, w_{j 1}\right]_{1}\left(b_{1}\right) & =l\left[\sum_{k=1}^{m_{1}} e_{i k} u_{k}, \sum_{s=1}^{m_{1}} e_{j s} u_{s}\right]_{1}\left(b_{1}\right) \\
& =l \sum_{k=1}^{m_{1}} \sum_{s=1}^{m_{1}} e_{i k} \bar{e}_{j s}\left[u_{k}, u_{s}\right]_{1}\left(b_{1}\right), \quad i, j=1, \ldots, d .
\end{aligned}
$$

Hence

$$
\left(l\left[w_{i 1}, w_{j 1}\right]_{1}\left(b_{1}\right)\right)_{d \times d}^{T}=\frac{1}{l} B_{1} U^{T} B_{1}^{*}=(-1)^{k} \frac{1}{l} B_{1} E_{m_{1}} B_{1}^{*},
$$

where the matrix $U$ is defined in Corollary 4.10. Note that $\left(E_{n}\right)^{*}=-E_{n},\left(E_{n}\right)^{-1}=-E_{n}$, and $A_{1}=(-1)^{k+1} l V_{1}^{*} E_{n}$, and we have

$$
\begin{aligned}
\left(l\left[w_{i 1}, w_{j 1}\right]_{1}\left(a_{1}\right)\right)_{d \times d}^{T} & =(-1)^{k} l V_{1}^{*} E_{n} V_{1}=(-1)^{k} l V_{1}^{*} E_{n} E_{n}^{-1} E_{n} V_{1} \\
& =(-1)^{k} l\left(V_{1}^{*} E_{n}\right)\left(-E_{n}\right)\left(-\left(E_{n}\right)^{*} V_{1}\right)=(-1)^{k} \frac{1}{l} A_{1} E_{n} A_{1}^{*} .
\end{aligned}
$$

Similarly,

$$
\begin{gathered}
\left(s\left[w_{i 2}, w_{j 2}\right]_{2}\left(b_{2}\right)\right)_{d \times d}^{T}=(-1)^{k} \frac{1}{s} B_{2} E_{m_{2}} B_{2}^{*}, \\
\left(s\left[w_{i 2}, w_{j 2}\right]_{2}\left(a_{2}\right)\right)_{d \times d}^{T}=(-1)^{k} \frac{1}{s} A_{2} E_{n} A_{2}^{*} .
\end{gathered}
$$

Hence condition (ii) of Lemma 4.11 becomes

$$
s A_{1} E_{n} A_{1}^{*}-s B_{1} E_{m_{1}} B_{1}^{*}+l A_{2} E_{n} A_{2}^{*}-l B_{2} E_{m_{2}} B_{2}^{*}=0 .
$$

Sufficiency. Let the matrices $A_{1}, B_{1}, A_{2}$ and $B_{2}$ satisfy the conditions (1) and (2). We prove that $D(S)$ defined by (3) is the domain of a self-adjoint extension $S$ of $S_{\min }$. Let

$$
\begin{gathered}
B_{1}=l\left(\bar{b}_{i j}\right)_{d \times m_{1}}, \quad(-1)^{k+1} E_{n} A_{1}^{*}=\bar{l}\left(\rho_{i j}\right)_{n \times d}, \\
s_{i 1}=\sum_{j=1}^{m_{1}} b_{i j} u_{j}, \quad i=1, \ldots, d .
\end{gathered}
$$

By the Naimark patching lemma, we may choose functions $w_{11}, \ldots, w_{d 1}$ in $D_{1 \max }$ such that

and

$$
w_{i 1}^{[j-1]}\left(a_{1}\right)=\rho_{i j}, \quad w_{i 1}^{[j-1]}(c)=s_{i 1}^{[j-1]}(c),
$$

$$
w_{i 1}(x)=s_{i 1}(x), \quad x \geq c,
$$

where $a_{1}<c<b_{1}, i=1, \ldots, d, j=1, \ldots, n$.

By (4.6) and (2.3),

$$
-A_{1}\left(\begin{array}{c}
y_{1}\left(a_{1}\right) \\
\vdots \\
y_{1}^{[n-1]}\left(a_{1}\right)
\end{array}\right)=(-1)^{k} l\left(\rho_{i j}\right)^{*} E_{n}\left(\begin{array}{c}
y_{1}\left(a_{1}\right) \\
\vdots \\
y_{1}^{[n-1]}\left(a_{1}\right)
\end{array}\right)=\left(\begin{array}{c}
l\left[y_{1}, w_{11}\right]_{1}\left(a_{1}\right) \\
\vdots \\
l\left[y_{1}, w_{d 1}\right]_{1}\left(a_{1}\right)
\end{array}\right) .
$$


By (4.7) and (4.8),

$$
B_{1}\left(\begin{array}{c}
{\left[y_{1}, u_{1}\right]_{1}\left(b_{1}\right)} \\
\vdots \\
{\left[y_{1}, u_{m_{1}}\right]_{1}\left(b_{1}\right)}
\end{array}\right)=\left(\begin{array}{c}
{\left[y_{1}, \bar{l} \sum_{j=1}^{m_{1}} b_{1 j} u_{j}\right]_{1}\left(b_{1}\right)} \\
\vdots \\
{\left[y_{1}, \bar{l} \sum_{j=1}^{m_{1}} b_{d j} u_{j}\right]_{1}\left(b_{1}\right)}
\end{array}\right)=\left(\begin{array}{c}
l\left[y_{1}, w_{11}\right]_{1}\left(b_{1}\right) \\
\vdots \\
l\left[y_{1}, w_{d 1}\right]_{1}\left(b_{1}\right)
\end{array}\right) .
$$

Similarly,

$$
\begin{gathered}
-A_{2}\left(\begin{array}{c}
y_{2}\left(a_{2}\right) \\
\vdots \\
y_{2}^{[n-1]}\left(a_{2}\right)
\end{array}\right)=\left(\begin{array}{c}
s\left[y_{2}, w_{12}\right]_{2}\left(a_{2}\right) \\
\vdots \\
s\left[y_{2}, w_{d 2}\right]_{2}\left(a_{2}\right)
\end{array}\right), \\
B_{2}\left(\begin{array}{c}
{\left[y_{2}, v_{1}\right]_{2}\left(b_{2}\right)} \\
\vdots \\
{\left[y_{2}, v_{m_{2}}\right]_{2}\left(b_{2}\right)}
\end{array}\right)=\left(\begin{array}{c}
s\left[y_{2}, w_{12}\right]_{2}\left(b_{2}\right) \\
\vdots \\
s\left[y_{2}, w_{d 2}\right]_{2}\left(b_{2}\right)
\end{array}\right) .
\end{gathered}
$$

Therefore the boundary condition (3) becomes the boundary condition (iii), that is,

$$
l\left[y_{1}, w_{i 1}\right]_{1}\left(b_{1}\right)-l\left[y_{1}, w_{i 1}\right]_{1}\left(a_{1}\right)+s\left[y_{2}, w_{i 2}\right]_{2}\left(b_{2}\right)-s\left[y_{2}, w_{i 2}\right]_{2}\left(a_{2}\right)=0, \quad i=1, \ldots, d .
$$

It remains to show that $w_{i}, i=1, \ldots, d$, satisfy the conditions (i) and (ii) of Lemma 4.11.

The condition (i) holds. If not, then there exist constants $c_{1}, \ldots, c_{d}$, not all zero, such that

$$
\gamma=\sum_{i=1}^{d} c_{i} w_{i} \in D_{\min }
$$

that is,

$$
\gamma_{1}=\sum_{i=1}^{d} c_{i} w_{i 1} \in D_{1 \min }, \quad \gamma_{2}=\sum_{i=1}^{d} c_{i} w_{i 2} \in D_{2 \min }
$$

Hence

$$
\left(\begin{array}{c}
\gamma_{1}\left(a_{1}\right) \\
\vdots \\
\gamma_{1}^{[n-1]}\left(a_{1}\right)
\end{array}\right)=\left(\rho_{i j}\right)_{n \times d}\left(\begin{array}{c}
c_{1} \\
\vdots \\
c_{d}
\end{array}\right)=(-1)^{k+1} \frac{1}{l} E_{n} A_{1}^{*}\left(\begin{array}{c}
c_{1} \\
\vdots \\
c_{d}
\end{array}\right)=\left(\begin{array}{c}
0 \\
\vdots \\
0
\end{array}\right) .
$$

Since $E_{n}$ is nonsingular and $l>0$, we conclude that

$$
\left(\bar{c}_{1} \cdots \bar{c}_{d}\right) A_{1}=0 .
$$

Similarly,

$$
\left(\bar{c}_{1} \cdots \bar{c}_{d}\right) A_{2}=0
$$

By (4.7) and (4.8),

$$
\gamma_{1}(x)=\sum_{i=1}^{d} c_{i} w_{i 1}(x)=\sum_{i=1}^{d} c_{i} \sum_{j=1}^{m_{1}} b_{i j} u_{j}(x), \quad x \geq c .
$$


Since $\gamma_{1} \in D_{1 \text { min }}$, it follows that $\left[\gamma_{1}, y_{1}\right]_{1}\left(b_{1}\right)=0$ for any $y=\left\{y_{1}, y_{2}\right\} \in D_{\max }$. Hence

$$
(0 \cdots 0)=\left(\left[\gamma_{1}, u_{1}\right]_{1}\left(b_{1}\right) \cdots\left[\gamma_{1}, u_{m_{1}}\right]_{1}\left(b_{1}\right)\right)=\left(c_{1} \cdots c_{d}\right)\left(b_{i j}\right)_{d \times m_{1}} U,
$$

Since $U$ is nonsingular, $\left(\bar{c}_{1} \cdots \bar{c}_{d}\right) B_{1}=0$.

Similarly, $\left(\bar{c}_{1} \cdots \bar{c}_{d}\right) B_{2}=0$.

Hence

$$
\left(\bar{c}_{1} \cdots \bar{c}_{d}\right)\left(A_{1}, B_{1}, A_{2}, B_{2}\right)=0 .
$$

This contradicts the fact that $\operatorname{rank}\left(A_{1}, B_{1}, A_{2}, B_{2}\right)=d$.

Next we show that (ii) holds. By (4.7) and (4.8),

$$
l\left[w_{i 1}, w_{j 1}\right]_{1}\left(b_{1}\right)=l\left[\sum_{s=1}^{m_{1}} b_{i s} u_{s}, \sum_{k=1}^{m_{1}} b_{j k} u_{k}\right]_{1}\left(b_{1}\right)=l \sum_{s=1}^{m_{1}} \sum_{k=1}^{m_{1}} b_{i s} \bar{b}_{j k}\left[u_{s}, u_{k}\right]_{1}\left(b_{1}\right) .
$$

Hence

$$
\left(l\left[w_{i 1}, w_{j 1}\right]_{1}\left(b_{1}\right)\right)_{d \times d}^{T}=\frac{1}{l} B_{1} U^{T} B_{1}^{*}=(-1)^{k} \frac{1}{l} B_{1} E_{m_{1}} B_{1}^{*} .
$$

Similarly,

$$
\left(s\left[w_{i 2}, w_{j 2}\right]_{2}\left(b_{2}\right)\right)_{d \times d}^{T}=\frac{1}{s} B_{2} V^{T} B_{2}^{*}=(-1)^{k} \frac{1}{s} B_{2} E_{m_{2}} B_{2}^{*} .
$$

Moreover,

$$
l\left(\left[w_{i 1}, w_{j 1}\right]_{1}\left(a_{1}\right)\right)_{d \times d}^{T}=(-1)^{k} l\left(\rho_{i j}\right)^{*} E_{n}\left(\rho_{i j}\right)_{n \times d}=(-1)^{k} \frac{1}{l} A_{1} E_{n} A_{1}^{*} .
$$

Similarly,

Therefore

$$
\left(s\left[w_{i 2}, w_{j 2}\right]_{2}\left(a_{2}\right)\right)_{d \times d}^{T}=(-1)^{k} \frac{1}{s} A_{2} E_{n} A_{2}^{*} .
$$

$$
\begin{gathered}
\left(l\left[w_{i 1}, w_{j 1}\right]_{1}\left(b_{1}\right)-l\left[w_{i 1}, w_{j 1}\right]_{1}\left(a_{1}\right)+s\left[w_{i 2}, w_{j 2}\right]_{2}\left(b_{2}\right)-s\left[w_{i 2}, w_{j 2}\right]_{2}\left(a_{2}\right)\right)^{T} \\
=(-1)^{k} \frac{1}{l} B_{1} E_{m_{1}} B_{1}^{*}-(-1)^{k} \frac{1}{l} A_{1} E_{n} A_{1}^{*} \\
\quad+(-1)^{k} \frac{1}{s} B_{2} E_{m_{2}} B_{2}^{*}-(-1)^{k} \frac{1}{s} A_{2} E_{n} A_{2}^{*}=0 .
\end{gathered}
$$

By Lemma 4.11, we conclude that $D(S)$ is a self-adjoint domain.

Remark 4.14. We call $u_{1}, \ldots, u_{m_{1}}$ and $v_{1}, \ldots, v_{m_{2}}$ LC solutions at $b_{1}$ and $b_{2}$, respectively. They are used to characterise the self-adjoint boundary conditions at the singular endpoints $b_{1}$ and $b_{2}$, while the remaining $d_{1}-m_{1}$ and $d_{2}-m_{2}$ LP solutions $u_{m_{1}+1}, \ldots, u_{d_{1}}$ and $v_{m_{2}+1}, \ldots, v_{d_{2}}$ do not contribute to the singular boundary conditions. In fact, for any $y=\left\{y_{1}, y_{2}\right\} \in D_{\max }$, we have $\left[y_{1}, u_{j}\right]_{1}\left(b_{1}\right)=0$, $j=m_{1}+1, \ldots, d_{1}$, and $\left[y_{2}, v_{j}\right]_{2}\left(b_{2}\right)=0, j=m_{2}+1, \ldots, d_{2}$.

In Theorem 4.13 it is assumed that the endpoints $a_{1}$ and $a_{2}$ are regular and $b_{1}, b_{2}$ are singular. The proof of Theorem 4.13 can easily be modified to prove analogues of Theorem 4.13 as long as at least one endpoint of each interval $\left(a_{1}, b_{1}\right),\left(a_{2}, b_{2}\right)$ is regular. Thus we have variants of Theorem 4.13 for $a_{1}$ singular, $b_{1}$ regular, $a_{2}$ singular, 
$b_{2}$ regular as well as for $a_{1}$ singular, $b_{1}$ regular, $a_{2}$ regular, $b_{2}$ singular and $a_{1}$ regular, $b_{1}$ singular, $a_{2}$ singular, $b_{2}$ regular. In the next theorem we state one of these cases explicitly as an illustration.

THeorem 4.15. Assume that $a_{1}$ is singular, $b_{1}$ is regular, $a_{2}$ is singular and $b_{2}$ is regular. Then a linear submanifold $D(S)$ of $D_{\max }$ is the domain of a self-adjoint extension $S$ of $S_{\text {min }}$ if and only if there exist a complex $d \times m_{1}$ matrix $A_{1}$, ad $d \times n$ matrix $B_{1}, a d \times m_{2}$ matrix $A_{2}$ and a $d \times n$ matrix $B_{2}$ such that the following three conditions hold.

(1) $\operatorname{rank}\left(A_{1}, B_{1}, A_{2}, B_{2}\right)=d$.

(2) $s A_{1} E_{m_{1}} A_{1}^{*}-s B_{1} E_{n} B_{1}^{*}+l A_{2} E_{m_{2}} A_{2}^{*}-l B_{2} E_{n} B_{2}^{*}=0$.

(3) $D(S)=\left\{y=\left\{y_{1}, y_{2}\right\} \in D_{\max }\right\}$, where

$$
\begin{aligned}
& A_{1}\left(\begin{array}{c}
{\left[y_{1}, u_{1}\right]_{1}\left(a_{1}\right)} \\
\vdots \\
{\left[y_{1}, u_{m_{1}}\right]_{1}\left(a_{1}\right)}
\end{array}\right)+B_{1}\left(\begin{array}{c}
y_{1}\left(b_{1}\right) \\
\vdots \\
y_{1}^{[n-1]}\left(b_{1}\right)
\end{array}\right) \\
& +A_{2}\left(\begin{array}{c}
{\left[y_{2}, v_{1}\right]_{2}\left(a_{2}\right)} \\
\vdots \\
{\left[y_{2}, v_{m_{2}}\right]_{2}\left(a_{2}\right)}
\end{array}\right)+B_{2}\left(\begin{array}{c}
y_{2}\left(b_{2}\right) \\
\vdots \\
y_{2}^{[n-1]}\left(b_{2}\right)
\end{array}\right)=\left(\begin{array}{c}
0 \\
\vdots \\
0
\end{array}\right) .
\end{aligned}
$$

In (2), $E_{j}$ is the symplectic matrix (2.2) of order $j$.

Proof. The proof is similar to that of Theorem 4.13 and therefore omitted.

\section{Examples}

To illustrate the self-adjoint boundary conditions given by Theorem 4.13 and its variants we give a number of examples. Here we give some examples for

$$
n=4, \quad 4 \leq d \leq 8 .
$$

Similar examples can easily be constructed for all higher-order cases $n=2 k, k>2$.

ExAMPLE 5.1. Assume the endpoint $a_{1}$ is singular, $b_{1}$ is regular, $a_{2}$ is regular and $b_{2}$ is singular. In the minimal deficiency case $d=4$, we have $d_{1}=2, d_{2}=2, m_{1}=2 d_{1}-4=$ $0, m_{2}=2 d_{2}-4=0$. Suppose that the boundary conditions at $b_{1}, a_{2}$ are coupled:

$$
\begin{gathered}
\left(\begin{array}{l}
y_{1}\left(b_{1}\right) \\
y_{1}^{[1]}\left(b_{1}\right) \\
y_{1}^{[2]}\left(b_{1}\right) \\
y_{1}^{[3]}\left(b_{1}\right)
\end{array}\right)=K\left(\begin{array}{l}
y_{2}\left(a_{2}\right) \\
y_{2}^{[1]}\left(a_{2}\right) \\
y_{2}^{[2]}\left(a_{2}\right) \\
y_{2}^{[3]}\left(a_{2}\right)
\end{array}\right), \\
K=\left(k_{i j}\right), \quad k_{i j} \in \mathbb{R}, i, j=1,2,3,4, \quad M_{14}-N_{14}<0, M_{23}-N_{23}>0, \\
M_{i j}=\left|\begin{array}{ll}
k_{i 2} & k_{i 3} \\
k_{j 2} & k_{j 3}
\end{array}\right|, \quad N_{i j}=\left|\begin{array}{ll}
k_{i 1} & k_{i 4} \\
k_{j 1} & k_{j 4}
\end{array}\right|, \quad i<j, i=1,2,3, j=2,3,4 .
\end{gathered}
$$


Let

$$
B_{1}=\left(\begin{array}{rrrr}
-1 & 0 & 0 & 0 \\
0 & -1 & 0 & 0 \\
0 & 0 & -1 & 0 \\
0 & 0 & 0 & -1
\end{array}\right), \quad A_{2}=K=\left(\begin{array}{llll}
k_{11} & k_{12} & k_{13} & k_{14} \\
k_{21} & k_{22} & k_{23} & k_{24} \\
k_{31} & k_{32} & k_{33} & k_{34} \\
k_{41} & k_{42} & k_{43} & k_{44}
\end{array}\right) \text {. }
$$

Then $\operatorname{rank}\left(B_{1}, A_{2}\right)=4$. From a straightforward computation, it follows that

$$
s B_{1} E B_{1}^{*}=l A_{2} E A_{2}^{*}, \quad E=\left(\begin{array}{rrrr}
0 & 0 & 0 & -1 \\
0 & 0 & 1 & 0 \\
0 & -1 & 0 & 0 \\
1 & 0 & 0 & 0
\end{array}\right)
$$

is equivalent to:

(1) $M_{12}=N_{12}$;

(2) $M_{13}=N_{13}$;

(3) $M_{24}=N_{24}$;

(4) $M_{34}=N_{34}$;

(5) $l\left(M_{14}-N_{14}\right)=-s$;

(6) $l\left(M_{23}-N_{23}\right)=s$.

Therefore, if $l=1$ and $s>0$ so $M_{14}-N_{14}=-s, M_{23}-N_{23}=s$, and (1), (2), (3), (4) are satisfied, then the boundary conditions (5.1) are self-adjoint.

Remark 5.2. Note that $s>0$ is needed to preserve the positivity of the inner product (3.2). Using appropriate multiples of the usual inner product, or changing the weight function $w_{2}$ to $s w_{2}$, we can generate self-adjoint operators for any real coupling matrix $K$ satisfying $M_{14}-N_{14}=-s<0, M_{23}-N_{23}=s>0$ and (1), (2), (3), (4). This contrasts with the results in [7], where using the weight function $w_{2}$ requires $M_{14}-N_{14}=-1, M_{23}-N_{23}=1$ and (1), (2), (3), (4) for self-adjointness. We see that the parameter $s$ plays a role in establishing the self-adjoint boundary conditions.

ExAmple 5.3. Let the endpoint $a_{1}$ be regular, $b_{1}$ singular, $a_{2}$ regular and $b_{2}$ singular. Assume $d_{1}=3, d_{2}=2$. Then $d=5$ and $m_{1}=2, m_{2}=0$. Consider a separated condition at $b_{1}$ and coupled conditions at $a_{1}, a_{2}$ :

$$
\begin{gathered}
C_{1}\left[y_{1}, u_{1}\right]_{1}\left(b_{1}\right)+C_{2}\left[y_{1}, u_{2}\right]_{1}\left(b_{1}\right)=0, \quad C_{1}, C_{2} \in R,\left(C_{1}, C_{2}\right) \neq(0,0), \\
\left(\begin{array}{l}
y_{2}\left(a_{2}\right) \\
y_{2}^{[1]}\left(a_{2}\right) \\
y_{2}^{[2]}\left(a_{2}\right) \\
y_{2}^{[3]}\left(a_{2}\right)
\end{array}\right)=K\left(\begin{array}{l}
y_{1}\left(a_{1}\right) \\
y_{1}^{[1]}\left(a_{1}\right) \\
y_{1}^{[2]}\left(a_{1}\right) \\
y_{1}^{[3]}\left(a_{1}\right)
\end{array}\right), \\
K=\left(k_{i j}\right), \quad k_{i j} \in \mathbb{R}, i, j=1,2,3,4, \quad M_{14}-N_{14}>0, M_{23}-N_{23}<0, \\
M_{i j}=\left|\begin{array}{ll}
k_{i 2} & k_{i 3} \\
k_{j 2} & k_{j 3}
\end{array}\right|, \quad N_{i j}=\left|\begin{array}{ll}
k_{i 1} & k_{i 4} \\
k_{j 1} & k_{j 4}
\end{array}\right|, \quad i<j, i=1,2,3, j=2,3,4 .
\end{gathered}
$$


Let

$$
A_{1}=K=\left(\begin{array}{cccc}
k_{11} & k_{12} & k_{13} & k_{14} \\
k_{21} & k_{22} & k_{23} & k_{24} \\
k_{31} & k_{32} & k_{33} & k_{34} \\
k_{41} & k_{42} & k_{43} & k_{44} \\
0 & 0 & 0 & 0
\end{array}\right), \quad B_{1}=\left(\begin{array}{ll}
0 & 0 \\
0 & 0 \\
0 & 0 \\
0 & 0 \\
c_{1} & c_{2}
\end{array}\right), \quad A_{2}=\left(\begin{array}{rrrr}
-1 & 0 & 0 & 0 \\
0 & -1 & 0 & 0 \\
0 & 0 & -1 & 0 \\
0 & 0 & 0 & -1 \\
0 & 0 & 0 & 0
\end{array}\right) .
$$

In this case $\operatorname{rank}\left(A_{1}, B_{1}, A_{2}\right)=5$ and $B_{1} E_{2} B_{1}^{*}=0$. Then in terms of Theorem 4.13, we obtain the equivalence of the conditions for self-adjointness:

(1) $M_{12}=N_{12}$;

(2) $M_{13}=N_{13}$;

(3) $M_{24}=N_{24}$;

(4) $M_{34}=N_{34}$;

(5) $s\left(M_{14}-N_{14}\right)=l$;

(6) $s\left(M_{23}-N_{23}\right)=-l$.

Therefore, if $s=1$ and $l>0$ so $M_{14}-N_{14}=l$ and $M_{23}-N_{23}=-l$, and (1), (2), (3), (4) are satisfied, then the boundary conditions (5.2) are self-adjoint.

Note that by studying the two-interval theory in direct-sum spaces with inner product multiples we obtain self-adjoint operators for any real coupling matrix $K$ satisfying $M_{14}-N_{14}=l>0, M_{23}-N_{23}=-l<0$ and (1), (2), (3), (4). This contrasts with the results in [7] which require $M_{14}-N_{14}=1, M_{23}-N_{23}=-1$ and (1), (2), (3), (4).

ExAmple 5.4. Assume $d_{1}=3, d_{2}=3$. Then $d=6$, and $m_{1}=2, m_{2}=2$. Let $a_{1}$ be regular, $b_{1}$ singular, $a_{2}$ singular and $b_{2}$ regular. Consider two pairs of coupled conditions:

$$
\begin{gathered}
\left(\begin{array}{l}
{\left[y_{2}, v_{1}\right]_{2}\left(a_{2}\right)} \\
{\left[y_{2}, v_{2}\right]_{2}\left(a_{2}\right)}
\end{array}\right)=G\left(\begin{array}{l}
{\left[y_{1}, u_{1}\right]_{1}\left(b_{1}\right)} \\
{\left[y_{1}, u_{2}\right]_{1}\left(b_{1}\right)}
\end{array}\right), \\
G=\left(\begin{array}{ll}
\left.g_{i j}\right), & g_{i j} \in \mathbb{R}, \quad i, j=1,2, \quad \operatorname{det} G>0, \\
y_{2}^{[1]}\left(b_{2}\right) \\
y_{2}^{[2]}\left(b_{2}\right) \\
y_{2}^{[3]}\left(b_{2}\right)
\end{array}\right)=K\left(\begin{array}{l}
y_{2}\left(b_{2}\right) \\
y_{1}^{[1]}\left(a_{1}\right) \\
y_{1}^{[2]}\left(a_{1}\right) \\
y_{1}^{[3]}\left(a_{1}\right)
\end{array}\right), \\
K=\left(k_{i j}\right), \quad k_{i j} \in \mathbb{R}, i, j=1,2,3,4, \quad M_{14}-N_{14}<0, M_{23}-N_{23}>0, \\
M_{i j}=\left|\begin{array}{ll}
k_{i 2} & k_{i 3} \\
k_{j 2} & k_{j 3}
\end{array}\right|, \quad N_{i j}=\left|\begin{array}{ll}
k_{i 1} & k_{i 4} \\
k_{j 1} & k_{j 4}
\end{array}\right|, \quad i<j, i=1,2,3, j=2,3,4 .
\end{gathered}
$$

Proceeding as in the previous example we obtain the equivalence of conditions for self-adjointness:

$$
s G E_{2} G^{*}=l E_{2} \quad \text { and } \quad s K E_{4} K^{*}=l E_{4},
$$


$s(\operatorname{det} G)=l$ and the following equations.

(1) $M_{12}=N_{12}$.

(2) $M_{13}=N_{13}$.

(3) $M_{24}=N_{24}$.

(4) $M_{34}=N_{34}$.

(5) $s\left(M_{14}-N_{14}\right)=-l$.

(6) $s\left(M_{23}-N_{23}\right)=l$.

If we choose $s=1$ and $l>0$ so $\operatorname{det} G=l>0$ and $M_{14}-N_{14}=-l<0, M_{23}-N_{23}=$ $l>0$, and (1), (2), (3), (4) are satisfied, then boundary conditions (5.3) and (5.4) are self-adjoint.

REMARK 5.5. Using appropriate multiples of the usual inner product, we establish self-adjoint operators for any real coupling matrix $K$ satisfying $\operatorname{det} G=l>0$ and $M_{14}-N_{14}=-l<0, M_{23}-N_{23}=l>0$ and (1), (2), (3), (4). This contrasts with the results in [7] which require $\operatorname{det} G=1$ and $M_{14}-N_{14}=-1, M_{23}-N_{23}=1$ and (1), (2), (3), (4).

Example 5.6. Assume $d_{1}=3, d_{2}=4$. Then $d=7$ and $m_{1}=2, m_{2}=4$. Let $a_{1}$ be regular, $b_{1}$ singular, $a_{2}$ regular and $b_{2}$ singular. Consider separated conditions at $a_{1}$ and at $b_{1}$ and coupled conditions at $a_{2}, b_{2}$ :

$$
\begin{gathered}
y_{1}\left(a_{1}\right)+i y_{1}^{[1]}\left(a_{1}\right)=0, \quad i y_{1}^{[2]}\left(a_{1}\right)+y_{1}^{[3]}\left(a_{1}\right)=0, \\
C_{1}\left[y_{1}, u_{1}\right]_{1}\left(b_{1}\right)+C_{2}\left[y_{1}, u_{2}\right]_{1}\left(b_{1}\right)=0, \quad C_{1}, C_{2} \in R,\left(C_{1}, C_{2}\right) \neq(0,0), \\
A_{2}\left(\begin{array}{l}
y_{2}\left(a_{2}\right) \\
y_{2}^{[1]}\left(a_{2}\right) \\
y_{2}^{[2]}\left(a_{2}\right) \\
y_{2}^{[3]}\left(a_{2}\right)
\end{array}\right)+B_{2}\left(\begin{array}{l}
{\left[y_{2}, v_{1}\right]_{2}\left(b_{2}\right)} \\
{\left[y_{2}, v_{2}\right]_{2}\left(b_{2}\right)} \\
{\left[y_{2}, v_{3}\right]_{2}\left(b_{2}\right)} \\
{\left[y_{2}, v_{4}\right]_{2}\left(b_{2}\right)}
\end{array}\right)=0 .
\end{gathered}
$$

Then $s A_{1} E_{4} A_{1}^{*}-s B_{1} E_{2} B_{1}^{*}=0$ for any $s$ since $A_{1} E_{4} A_{1}^{*}=0=B_{1} E_{2} B_{1}^{*}$. In terms of Theorem 4.13, the boundary conditions (5.5) and (5.6) are self-adjoint if and only if $\operatorname{rank}\left(A_{2}, B_{2}\right)=4$ and

$$
A_{2} E_{4} A_{2}^{*}-B_{2} E_{4} B_{2}^{*}=0 \text {. }
$$

Note that these conditions are independent of $l$ and $s$ and are simply the one-interval self-adjointness conditions for each of the two intervals separately. Thus the above example just gives the two-interval self-adjointness conditions which are generated by the direct sum of self-adjoint operators from each of the two intervals separately.

ExAmple 5.7. Assume $a_{1}$ is regular, $b_{1}$ is singular, $a_{2}$ is regular and $b_{2}$ is singular. In the maximal deficiency case $d=8$, we have $d_{1}=4, d_{2}=4$ and $m_{1}=4, m_{2}=4$. 
Consider separated conditions at $a_{1}$ and at $a_{2}$ and coupled conditions at $b_{1}, b_{2}$ :

$$
\begin{gathered}
\left\{\begin{array}{l}
y_{1}\left(a_{1}\right)+i y_{1}^{[1]}\left(a_{1}\right)=0, i y_{1}^{[2]}\left(a_{1}\right)+y_{1}^{[3]}\left(a_{1}\right)=0, \\
y_{2}\left(a_{2}\right)+i y_{2}^{[1]}\left(a_{2}\right)=0, i y_{2}^{[2]}\left(a_{2}\right)+y_{2}^{[3]}\left(a_{2}\right)=0,
\end{array}\right. \\
\left(\begin{array}{l}
{\left[y_{1}, u_{1}\right]_{1}\left(b_{1}\right)} \\
{\left[y_{1}, u_{2}\right]_{1}\left(b_{1}\right)} \\
{\left[y_{1}, u_{3}\right]_{1}\left(b_{1}\right)} \\
{\left[y_{1}, u_{4}\right]_{1}\left(b_{1}\right)}
\end{array}\right)=K\left(\begin{array}{l}
{\left[y_{2}, v_{1}\right]_{2}\left(b_{2}\right)} \\
{\left[y_{2}, v_{2}\right]_{2}\left(b_{2}\right)} \\
{\left[y_{2}, v_{3}\right]_{2}\left(b_{2}\right)} \\
{\left[y_{2}, v_{4}\right]_{2}\left(b_{2}\right)}
\end{array}\right), \\
K=\left(k_{i j}\right), \quad k_{i j} \in \mathbb{R}, i, j=1,2,3,4, \quad M_{14}-N_{14}>0, M_{23}-N_{23}<0, \\
M_{i j}=\left|\begin{array}{ll}
k_{i 2} & k_{i 3} \\
k_{j 2} & k_{j 3}
\end{array}\right|, \quad N_{i j}=\left|\begin{array}{ll}
k_{i 1} & k_{i 4} \\
k_{j 1} & k_{j 4}
\end{array}\right|, \quad i<j, i=1,2,3, j=2,3,4 .
\end{gathered}
$$

Let

$$
\begin{aligned}
& A_{1}=\left(\begin{array}{llll}
0 & 0 & 0 & 0 \\
0 & 0 & 0 & 0 \\
0 & 0 & 0 & 0 \\
0 & 0 & 0 & 0 \\
1 & i & 0 & 0 \\
0 & 0 & i & 1 \\
0 & 0 & 0 & 0 \\
0 & 0 & 0 & 0
\end{array}\right), \quad B_{1}=\left(\begin{array}{rrrr}
-1 & 0 & 0 & 0 \\
0 & -1 & 0 & 0 \\
0 & 0 & -1 & 0 \\
0 & 0 & 0 & -1 \\
0 & 0 & 0 & 0 \\
0 & 0 & 0 & 0 \\
0 & 0 & 0 & 0 \\
0 & 0 & 0 & 0
\end{array}\right), \\
& A_{2}=\left(\begin{array}{cccc}
0 & 0 & 0 & 0 \\
0 & 0 & 0 & 0 \\
0 & 0 & 0 & 0 \\
0 & 0 & 0 & 0 \\
0 & 0 & 0 & 0 \\
0 & 0 & 0 & 0 \\
1 & i & 0 & 0 \\
0 & 0 & i & 1
\end{array}\right), \quad B_{2}=\left(\begin{array}{cccc}
k_{11} & k_{12} & k_{13} & k_{14} \\
k_{21} & k_{22} & k_{23} & k_{24} \\
k_{31} & k_{32} & k_{33} & k_{34} \\
k_{41} & k_{42} & k_{43} & k_{44} \\
0 & 0 & 0 & 0 \\
0 & 0 & 0 & 0 \\
0 & 0 & 0 & 0 \\
0 & 0 & 0 & 0
\end{array}\right) .
\end{aligned}
$$

In this case $\operatorname{rank}\left(A_{1}, B_{1}, A_{2}, B_{2}\right)=8$ and $s A_{1} E A_{1}^{*}+l A_{2} E A_{2}^{*}=0$ for any $s, l$ since $A_{1} E A_{1}^{*}=0=A_{2} E A_{2}^{*}$. Therefore the boundary conditions (5.7) and (5.8) are selfadjoint if and only if:

(1) $M_{12}=N_{12}$;

(2) $M_{13}=N_{13}$;

(3) $M_{24}=N_{24}$;

(4) $M_{34}=N_{34}$;

(5) $l\left(M_{14}-N_{14}\right)=s$;

(6) $l\left(M_{23}-N_{23}\right)=-s$. 
If we choose $l=1$ and $s>0$ so $M_{14}-N_{14}=s>0$ and $M_{23}-N_{23}=-s<0$, and (1), (2), (3), (4) are satisfied, then the boundary conditions (5.7) and (5.8) are self-adjoint. Note that the matrices generating this self-adjoint operator are nonreal.

\section{References}

[1] J. P. Boyd, 'Sturm-Liouville eigenvalue problems with an interior pole', J. Math. Phys. 22(8) (1981), 1575-1590.

[2] W. N. Everitt and A. Zettl, 'Sturm-Liouville differential operators in direct sum spaces', Rocky Mountain J. Math. 16(3) (1986), 497-516.

[3] W. N. Everitt and A. Zettl, 'Differential operators generated by a countable number of quasidifferential expressions on the line', Proc. Lond. Math. Soc. (3) 64 (1992), 524-544.

[4] F. Gesztesy and W. Kirsch, 'One-dimensional Schrödinger operators with interactions singular on a discrete set', J. reine angew. Math. 362 (1985), 28-50.

[5] X. Hao, J. Sun, A. Wang and A. Zettl, 'Characterization of domains of self-adjoint ordinary differential operators $\amalg '$, Results Math., to appear.

[6] O. S. Mukhtarov and S. Yakubov, 'Problems for differential equations with transmission conditions', Appl. Anal. 81 (2002), 1033-1064.

[7] J. Q. Suo and W. Y. Wang, 'Two-interval even order differential operators in direct sum spaces', Results Math., to appear.

[8] A. P. Wang, J. Sun and A. Zettl, 'Two-interval Sturm-Liouville operators in modified Hilbert spaces', J. Math. Anal. Appl. 328 (2007), 390-399.

[9] A. Wang, J. Sun and A. Zettl, 'Characterization of domains of self-adjoint ordinary differential operators', J. Differential Equations 246 (2009), 1600-1622.

[10] A. Zettl, Sturm-Liouville Theory, Mathematical Surveys and Monographs, 121 (American Mathematical Society, Providence, RI, 2005).

JIANQING SUO, Math. Dept., Inner Mongolia University,

Hohhot, 010021, China

e-mail: sjq.hello@163.com

WANYI WANG, Math. Dept., Inner Mongolia University,

Hohhot, 010021, China

e-mail: wwy@imu.edu.cn 